\title{
Selection-free non-viral method revealed highly efficient CRISPR-Cas9 genome editing of human pluripotent stem cells guided by cellular autophagy
}

\author{
Michelle Surma ${ }^{1}$, Kavitha Anbarasu ${ }^{1,2}$ and Arupratan Das Da, $^{1,3,4,5, *}$
}

${ }^{1}$ Department of Ophthalmology, Eugene and Marilyn Glick Eye Institute, Indiana University, Indianapolis, IN 46202, USA; ${ }^{2}$ Department of Medical and Molecular Genetics, Indiana University, Indianapolis, IN 46202, USA; ${ }^{3}$ Stark Neurosciences Research Institute, Indiana University, Indianapolis, IN 46202, USA; ${ }^{4}$ Department of Biochemistry and Molecular Biology, Indiana University, Indianapolis, IN 46202, USA

${ }^{5}$ Lead Contact

*Correspondence: arupdas@iu.edu

\section{SUMMARY}

CRISPR-Cas9 mediated genome editing of human pluripotent stem cells (hPSCs) provides strong avenues for human disease modeling, drug discovery and cell replacement therapy. Genome editing of hPSCs is an extremely inefficient process and requires complex gene delivery and selection methods to improve edit efficiency which are not ideal for clinical applications. Here, we have shown a selection free simple lipofectamine based transfection method where a single plasmid encoding guide RNA (gRNA) and Cas9 selectively transfected hPSCs at the colony edges. Upon dissection and sequencing, the edge cells showed more than $30 \%$ edit frequency compared to the reported $3 \%$ rate under no selections. Increased cellular health of the edge cells as revealed by reduced autophagy gene-expressions is critical for such transfection pattern. Edge specific transfection was inhibited by blocking lysosomal activity which is essential for autophagy. Hence, our method provides robust scarless genome-editing of hPSCs which is ideal for translational research. 


\section{INTRODUCTION}

Genome editing of hPSCs by CRISPR-Cas9 provides many unprecedented advantages,

34 from introducing disease specific gene deletions to precise DNA base pair changes.

Genome edited hPSCs are differentiated to the human cell of interest for disease

modeling, drug screening, or cell replacement therapy, with potential use for personalized

medicine (Saha and Jaenisch, 2009). Successful delivery of Cas9 gene and gRNA

specific to the target gene is critical for gene editing in hPSCs. However, CRISPR-Cas9

mediated genome editing of hPSCs is an extremely inefficient process with success rate

less than 3\% (Yang et al., 2013). To increase genome-editing efficiency in hPSCs, several

approaches have been taken including: stable integration of a drug selection marker into the genome (Lombardo et al., 2007), transient selection (Sluch et al., 2018; Steyer et al., 2018), fluorescence-activated cell sorting (FACS) (Ding et al., 2013) and more recently a combination of electroporation and viral transduction based methods (Martin et al., 2019). While these methods have improved genome editing efficiency, they also possess unwanted consequences, such as permanent gene alterations through integration of selection markers which could disrupt the local transcriptional regulation and will make

48 hPSCs incompatible for clinical applications. FACS based single cell sorting to enrich Cas9 expressing cells increased edit efficiency to $6.0 \%$ in hPSCs but with very low cell survival rate (Byrne and Church, 2015; Yang et al., 2013). Viral gene delivery methods require special skill in producing virus particles, which is a lengthy process and not ideal

52 for most laboratories. Antibiotic selection to enrich the transfected cells activates innate 53 immunity and genetic changes which are not ideal for downstream translational 
54 applications (Mignon et al., 2015; Vandermeulen et al., 2011). Thus, there is a critical need for developing a CRISPR-Cas9 mediated genome editing technique for hPSCs without the need for any antibiotic selection, FACS sorting or complex viral transduction-

57 based methods.

To achieve this goal, here we first explored the differential transfection potential of stem cells within a hPSC colony and have identified that cells at the colony edges selectively got transfected due to increased cellular health compared to the cells at the center. Using a single plasmid containing gRNA/Cas9 and lipofectamine based transfection, we found very high Cas 9 expression with more than $30 \%$ genome editing frequency through nonhomologous end joining (NHEJ) at the edge cells compared to the center. This simple but highly efficient scarless genome editing technique of hPSCs is ideal for disease modeling research and clinical applications.

\section{RESULTS}

We used a simple lipofectamine-based transfection to hPSCs which requires only mixing

73 of the lipofectamine reagent and plasmid DNA containing Cas9/gRNA followed by addition to the cells. To identify if hPSC culture shows any cellular pattern for transfection which could be exploited to enrich the transfected cells, first we transfected $\mathrm{H} 7$ human 
77 the colony stage which is formed by compacting several cells. Much to our surprise, we observed stem cells at the colony edge got selectively transfected but not cells the at colony center (Figure 1A), while single cell culture got transfected randomly with no such pattern (Figure 1B) as observed by the mCherry fluorescence. However, overall transfected cell population showed no difference between the single cell and colony stages when measured by flow cytometer (Figures 1C,1D). To measure if stem cells at the colony center are not expressing mCherry and hence not transfected, we drew a line across the colony center through the edges and measured the fluorescence intensity profile on that line (Figure 1E). Indeed, we observed specific fluorescence intensity peaks on the line corresponding to the edges but not at the center (Figure 1F). This observation was further verified by measuring fluorescence intensity around the colony edges and centers which showed significantly high expression at the edge but not at the center cells (Figure 1G). To test if selective transfection of the colony edge cells is a cell type specific phenomenon, we transfected human H9-ESCs and induced pluripotent stem cell (EP1iPSCs) (Bhise et al., 2013) colonies with CAG-mCherry plasmid. Indeed, we observed

92 increased transfection of the peripheral cells for both the hPSC lines (Figure S1A, S1D). This observation was further verified by measuring fluorescence intensity profiles across

94 the line through the colony center (Figure S1A, S1D) which showed specific intensity peaks at the colony edges but not at the center (Figure S1B, S1E). Similarly, fluorescence intensity measurements showed significantly high fluorescence at the edges compared

97 to centers (Figure S1C, S1F). As hPSCs could be maintained in various media which could affect transfection efficiency, we tested the two most commonly used stem cell media mTeSR1 (mT) and mTeSRplus (mTp) for their effect on hPSC colony transfection 
100 to further identify the optimum media for transfection. We found significantly higher 101 transfection of H7-ESC colonies when cultured in the $\mathrm{mT}$ compared to the mTp media

102 (Figure S2A, S2B) and maintained hPSCs in the mT for this study. However, cellular 103 stemness marker expressions such as OCT4, NANOG, SSEA1, SSEA4, Alkaline 104 Phosphatase (ALPL), SOX2, NOTCH1 and NESTIN did not alter between mT and mTp 105 media (Figure S2C) suggesting stemness property of hPSCs was unaffected when 106 cultured in either media. To test if selective transfection of the colony edge cells is specific 107 to the lipofectamine based transfection, we transduced H7-ESC colonies with the 108 lentivirus containing GFP vector. Interestingly, we observed stem cells throughout the 109 colony got transduced as shown by GFP expression losing the morphological pattern 110 (Figure S3). These data suggest lipofectamine based simple transfection of hPSC 111 colonies selectively transfects edge cells, which could then be isolated to enhance 112 CRISPR genome editing without the need of any viral transduction and antibiotic selection 113 methods.

Autophagy driven increased cellular health of hPSC colony edges caused increased cell transfection.

118 To investigate the mechanism of selective transfection of hPSC colony edge cells, we 119 asked if cells at the colony edges have more access to the nutrients from the media than 120 the cells at the center, leading to improved cellular health and transfection. Cellular health 121 could be measured by gene expression of the autophagy pathway genes as they get 122 upregulated under stress (Kroemer et al., 2010). Activation of the autophagy pathway 
123 genes help by removing damaged proteins and organelles under cellular stress (Anding and Baehrecke, 2017). Hence, we expect cells at the hPSC colony centers will have more autophagy gene expression than the edges. To measure this, we dissected out the H7hESC cells from the colony edges and centers (Figure 2A) and measured gene expressions for a broad range of autophagy genes (Sha et al., 2018). Indeed, we found key autophagy genes such as ATG5, LC3B, GABARAP, GABARAPL1 and ATG13 are upregulated at the center compared to the edge cells (Figure 2B) suggesting improved cellular health of the edge cells. Of note, the dissected center portion of colony (Figure 2A) also contain small portion of edges as dissection of only center cells are extremely difficult and growing colonies very large leads to colony fusions losing edge populations and spontaneous differentiation (Chen et al., 2014). Next, we asked whether the difference in cellular health between colony edge and center affects hPSC stemness property. To test this, we measured stemness marker gene expressions between the edge and center cells of H7-hESC colonies and found no significant difference (Figure 2C), suggesting stem cells maintained their stemness property throughout the colony. If stem cells at the colony edges show increased health due to the greater exposure to nutrients leading to more transfection, we asked if creating new edges at the colony center would lead to selective transfection of stem cells at the newly formed edges. To test this, 141 we scratched at the center of H7-hESC colonies to form new edges (Figure S4A) followed 142 by transfection using CAG-mCherry plasmid. Indeed, we observed that cells at the newly 143 formed edges at the former colony center got transfected as shown by the mCherry 144 expression (Figure S4B). Of note, we also have seen some stem cell transfection inside 145 these colony centers (Figure S4B), presumably due to the change in cellular contact upon 
scratching through the middle of colonies, which is a mechanical perturbation. These suggest that stem cells at the colony centers maintained their stemness as well as the ability to get transfected upon exposure to new edges.

It has been shown that cells at the hPSC colony edges experience strong myosin II molecular motor mediated contractility of the actin cytoskeleton leading to enhanced contraction of extracellular matrix (ECM) (Närvä et al., 2017; Rosowski et al., 2015). We asked if increased actomyosin contractility at the hPSC colony edges are responsible for selective transfection of these cells. To test this, we inhibited actomyosin contractility by the very potent myosin II ATPase inhibitor blebbistatin (Das et al., 2016) followed by transfection of H7-hESC colonies using CAG-mCherry plasmid. Interestingly, we observed that under myosin II inhibition cells at the edge as well as at the colony center got transfected, losing the edge specific transfection pattern as shown by mCherry expression (Figure 3A). We further observed an overall higher percentage of transfection under myosin II inhibition as measured by flow cytometry (Figure 3B, 3C) which also agrees with the previously reported data (Yen et al., 2014). Myosin inhibition presumably reduced cell-cell contact, making cells more exposed to the transfection reagent and causing cell transfection throughout the colony. Actomyosin contractility leads to the formation of thick F-actin stress fibers within the cells (Tojkander et al., 2012) which we observed in the edge cells of H7-ESC colonies with F-actin stained by fluorescence conjugated phalloidin, indicated by arrows (Figure 3D). We found successful inhibition of actomyosin contractility by blebbistatin as stress fibers disappeared at the hPSC colony edge cells (Figure 3D). Our data showed cells at the hPSC colony centers are under 
stress with increased autophagy gene expressions compared to the edges. We asked if inhibition of the autophagy pathway would increase cellular stress throughout the hPSC colony leading to the inhibition of edge specific cell transfection. To test this, we inhibited the autophagy pathway by using a lysosome inhibitor bafilomycin, followed by CAGmCherry plasmid transfection. Indeed, we found loss of colony edge specific transfection (Figure 3A) with reduced overall transfection under autophagy inhibition (Figure 3B, 3C) but no loss of F-actin stress fibers at the edge cells was observed (Figure 3D). These data suggest increased cellular health but not the actomyosin contractility of the hPSC colony edge cells is responsible for the selective transfection of those cells.

\section{Edge cells at the hPSC colonies showed very high efficiency CRISPR genome editing.}

Finally, we asked if selective transfection of the hPSC colony edge cells could be exploited to have high-efficient CRISPR-Cas9 genome editing. To test this, we transfected $\mathrm{H} 7-\mathrm{hESC}$ colonies using lipofectamine and a plasmid encoding gRNA under U6 promoter and SpCas9-2A-GFP under CAG promoter (Figure 4A). 2A is a nontranslatable sequence (Sharma et al., 2012) and after translation, SpCas9 and GFP remain separate without effecting the protein activity. We hypothesized selective transfection of the hPSC colony edge cells would lead to enhanced Cas9 expression in those cells. To test this, we transfected H7-hESC colonies with the above plasmid and dissected out colony edge and center as shown in Figure 4B and measured Cas9 expression in the respective populations. Indeed, we observed several fold increased 
192 Cas9 expression in edge cells compared to the center (Figure 4C). This is very important 193 as Cas9 delivery followed by gRNA-guided DNA double-strand break (DSB) leads to the 194 insertion-deletions (INDELs) which is the rate-limiting step for obtaining high-frequency 195 genome editing (Hendel et al., 2014, 2015).

197 Next, we cloned gRNA into the Cas9 vector (Figure 4A) for mutating hypoxanthine phosphoribosyltransferase 1 (HPRT1). Upon transfection of the H7-hESCs colonies with this plasmid, we observed selective transfection of the cells at the colony edge by GFP expression (Figure 4D) similar to the CAG-mCherry plasmid. gRNA targets a specific gene sequence which allows the Cas9 enzyme to bind and create DNA DSB which cells repair by NHEJ. NHEJ leads to INDELs causing gene mutations. These mutations could be detected by PCR amplifying the DNA sequence around the gRNA target site followed by sanger sequencing and TIDE analysis (Brinkman et al., 2014) of the sequencing data. Since we observed high Cas9 expression in the edge cells, we hypothesized this will lead to high INDEL frequency. Indeed, by TIDE analysis we observed $\sim 38 \%$ of edge cells with mutations (62\% at 0 INDEL corresponds to $38 \%$ mutation) for HPRT1-gene in comparison to the center cells (Figure 4E, F). This is a significant improvement from the reported $3 \%$ 209 mutation rate of hPSCs under non-viral and selection free conditions (Yang et al., 2013).

210 This is remarkable as for the first time it allowed us to identify cells from stem cell colonies 211 with very high-frequency genome editing without the need for any viral transduction, 212 FACS sorting or antibiotic selections. 
Our work here demonstrated properties of hPSCs within a colony and how that could be used to achieve a selection and viral transduction free CRISPR-Cas9 genome editing

218 technique with very high edit-frequency. This work will have three major impacts on the human disease modeling research; (1) the absence of any antibiotic selection marker will avoid integration of the marker gene and Cas9 into the hPSC genome avoiding unwanted scars or changes, (2) the simple lipofectamine reagent based transfection would allow us to use two or more plasmids with gRNAs targeted for different genes to have double or triple gene knock-out simultaneously, (3) this technique could also be used to create disease causing DNA base pair changes (point mutations) or correct mutations in the 225 patient derived iPSCs by using Cas9 plasmid and single-stranded oligodeoxynucleotides (ssODNs) donor or donor vector. The lipofectamine-stem reagent is compatible for

227 transfecting hPSCs with plasmids as well as ssODNs. Being able to seamlessly edit hPSCs would allow us to differentiate genome edited stem cells to the cell of interest and investigate the human disease mechanism, perform drug screening to identify cell protective agents and replace damaged cells with healthy cells in in-vivo models for cell replacement therapy. This will bring a paradigm shift in the understanding of genotype232 phenotype relationship for a variety of human diseases.

Our data suggested selective transfection of the colony edge cells is due to improved cellular health as revealed by low autophagy gene expressions compared to the center cells (Figure 2). However, it is also possible that the edge cells are more exposed to the

237 transfection reagents than the center leading to increased transfection. This notion could 
238 be supported by our colony scratch experiment where cells at the new edge in the middle 239 of hPSC colonies got transfected (Figure S4). Our data (Figure 3C) as well as data from

240 another group (Yen et al., 2014) have shown inhibiting actomyosin contractility increased

241 hPSC colony transfection, which could be due to the reduced cell-cell contact within the

242 colony centers exposing cells more to the transfection reagents. Thus, selective

243 transfection of the hPSC colony edge cells could be due to the combination of increased

244 cellular health and more exposure to the transfection reagents.

245

246

\section{Limitations of Study}

247 This study reveals a very simple but high-efficient genome editing technique in hPSCs

248 with tremendous potential for a broad range of gene editing applications. As a next step,

249 this technique could be used for introducing point mutations in hPSCs or correcting

250 mutations in patient derived iPSCs. Our method here relies on the compact hPSC colony

251 formation to have the distinct edge and center cell population. hPSCs typically grow

252 forming these compact colonies; however if stem cells are grown in non-colony type

253 monolayer (NCM) (Chen et al., 2012) they will not have distinct edge and center cell

254 populations, and hence will limit this method application.

255

256 ACKNOWLEDGEMENTS

257

258 This work was supported by the grant from the NIH, United States (R00EY028223). We

259 thank Dr. Donald Zack for kindly providing the H7-hESC, H9-hESC and EP1-iPSC human 
260 pluripotent stem cell lines and Drs. Jason Meyer, David Wallace and Timothy Corson for 261 valuable discussions.

\section{AUTHOR CONTRIBUTIONS}

M.S. and A.D. designed the experiments, analyzed data and wrote manuscript; M.S. supervised the project and revised the manuscript.

\section{DECLARATION OF INTERESTS}

271 The authors declare no competing interests

273 FIGURE LEGENDS

Figure 1: hESCs selectively got transfected at the colony edges but not at center.

275 H7-hESCs after clump or single cell passage were transfected with CAG-mCherry (red

276 fluorescence protein, RFP) plasmid and representative brightfield and RFP images were

277 taken $24 \mathrm{~h}$ after transfection. Shown are images of colony (A) and single cells (B). (C, D)

278 Transfected cells were dissociated by accutase and run through flow cytometer; shown

279 are the distribution of the RFP-positive cells (C) and quantification for percentage of total

280 RFP-positive cells (D) with 3 biological repeats for each condition. (E-F) Representative

281 images of clump passaged colony $24 \mathrm{~h}$ after transfection with CAG-mCherry plasmid (E),

282 line trace through the center of colony as shown in $(E)$ shows fluorescence intensity peaks 
283 at the edges (F) but not at the center. (G) Quantification of the fluorescence intensity of colony edges and centers from 58 colonies from 5 independent experiments. Error bars are SEM, Student's t-test, ***, p $<0.0005$.

Figure 2: hPSCs at the colony edges are healthier with reduced expression of autophagy genes.

(A) H7-hESCs were cultured and colony edges and centers were dissected for qPCR. (B,

C) qPCR analysis was done on the autophagy genes (B) and stemness marker genes center. Error bars are SEM, Student's t-test, *, p-value $<0.05, \mathrm{n}=7-15$.

Figure 3: Inhibiting autophagy but not actomyosin contractility decreases transfection efficiency.

(A) H7-hESC colonies were treated for $16 \mathrm{~h}$ with blebbistatin or bafilomycin, then Single cell solutions were collected $24 \mathrm{~h}$ after transfection and run through flow cytometer, control (C). Error bars are SEM, One-way Anova with Dunnett's post hoc; **, p-value < 0.001, n=3. (D) Shown are representative confocal immunofluorescence images of Factin labelled with Alexa Fluor 488 Phalloidin and nucleus labeled with DAPI of H7-hESCs

303 treated for $24 \mathrm{~h}$ with the indicated drugs. Arrows indicate normal actin stress fiber bundles, $304 n=12$. 
Figure 4: Enhanced CRISPR-Cas9 genome editing at the hPSC colony edges.

(A) Map of plasmid (Addgene \#79144) containing Cas9 and GFP cassettes. (B, C) H7hESCs were transfected with the above plasmid, edges and centers were dissected after as $\triangle \mathrm{Ct}$ fold change relative to GAPDH. Error bars are SEM, Student's $t$-test, ***, $\mathrm{p}$-value $<0.0005, \mathrm{n}=6-9$. (D-F) HPRT1-gRNA was inserted into the Addgene plasmid and then

312 transfected into $\mathrm{H} 7$-hESCs, shown are representative images after $24 \mathrm{~h}$ of transfection 313 (D). Colony edges and centers were dissected and sequenced for HPRT1 mutation, 314 shown are representative sequencing chromatographs (E) and INDEL distribution of edge 315 cells compared to the center cells by TIDE analysis (F), $n=12$.

STAR METHODS

Resource Availability

\section{Lead Contact}

322 Further information and requests for resources and reagents should be directed to

\section{Materials Availability}

327 Stem cells and plasmids are available from the Lead Contact's laboratory upon request and completion of the Material Transfer Agreement. 
This study did not generate any code or dataset.

EXPERIMENTAL MODEL AND SUBJECT DETAILS:

H7-ESCs, H9-ESCs (WiCell, https://www.wicell.org/), and EP1-iPSCs were grown in using Gentle Cell Dissociation Reagent (GD) after reaching 80\% confluency. GD was added to cells for 4 min at $37^{\circ} \mathrm{C}$, aspirated, then $\mathrm{mT}$ was used to resuspend colonies; cell suspension was mixed by pipetting 3-4 times to break up the colonies into small clumps and then seeded into new MG coated wells. Clump passaged colonies were cultured for with accutase for $10 \mathrm{~min}$ and then quenched with double volume of $\mathrm{mT}$ with $5 \mu \mathrm{M}$ 
352 hPSCs were cultured as described above. The clump passaged colonies using GD were 353 added to a larger volume of media and equally split into the wells of 24-well plates. Single cells after accutase passage were transfected $24 \mathrm{~h}$ after seeding. GD cells were cultured

355 for another 2-3 days until the colonies were established with distinct edges and centers with colony size around $1 / 10^{\text {th }}$ the size of a $10 x$ viewscreen at start of drug treatment or

357 transfection. hPSC colonies were treated with $5 \mu \mathrm{M}$ blebbistatin, $50 \mathrm{nM}$ bafilomycin, or equivalent volume of DMSO in $\mathrm{mT}$ for $16 \mathrm{~h}$. Cell transfections were done by mixing $2 \mu \mathrm{l}$ of lipofectamine stem (Invitrogen) and $600 \mathrm{ng}$ of indicated plasmids in $50 \mu \mathrm{l}$ optimem by vortex. $10 \mathrm{~min}$ after vortexing, this mixture was added to the cell culture and incubated for 24h. Images were taken by the EVOS fluorescence microscope (Thermo Fisher Scientific). Using ImageJ software, fluorescence intensity was quantified by drawing a 'donut' containing the colony edge, measured as the edge; the 'hole' of the donut was then measured as the center. Raw integrated density was divided by the total area to get the average intensity per area for both edge and center of each colony.

367 For flow cytometry, cells were incubated in $40 \mu \mathrm{l}$ accutase for $10 \mathrm{~min}$, then quenched with $368160 \mu \mathrm{mT}$ with $5 \mu \mathrm{M}$ blebbistatin. This $200 \mu \mathrm{l}$ cell suspension was transferred into a 96well round-bottom plate and read on the Attune NxT Acoustic Focusing Flow Cytometer

370 (Thermo) equipped with Attune Auto Sampler (Thermo). Gating was used first to separate

371 live cells, then to separate RFP-positive from the live cell population using the Attune NxT

372 Software. Data were exported to excel or prism for analysis and plotting. Three or more 373 biological repeats were performed for each condition. 
375 hPSCs were grown in mT and clump passaged using GD. For mT and mTp comparison, 376 H7-hESCs were cultured in the respective media in 6-well plates for more than 2 weeks 377 before starting the experiment. Cells at $\sim 80 \%$ confluency were passaged and seeded on 378 24-well MG coated plates for another 4-5 days until they reached $\sim 70 \%$ confluency. Cells were incubated with $200 \mu \mathrm{l}$ accutase for $10 \mathrm{~min}$ and resuspended in $400 \mu \mathrm{mT}$ with $5 \mu \mathrm{M}$ blebbistation. Cells were then centrifuged at $150 \mathrm{G}$ for 5 min, media aspirated, and cell pellets stored at $-20^{\circ} \mathrm{C}$. For edge/center comparison, colony dissection was done two days after seeding using clump passaging. When colonies were grown to $1 / 4$ of a $10 x$ field size checked by EVOS, the edge was dissected out first, and then a slice from the center of the colony was collected as the center. Samples were collected into $\mathrm{mT}$ with 5 $\mu \mathrm{M}$ blebbistatin, with edges and centers from 10 distinct colonies collected into 1 then centrifuged, media aspirated, and cell pellets stored at $-20^{\circ} \mathrm{C}$ until cDNA preparation. RNA extraction was done following the kit (Qiagen 74104) and $6 \mu$ l of RNA was used to prepare cDNA (abm G492). cDNA concentration was measured using Nanodrop 2000c (Thermo) and stored at $-80^{\circ} \mathrm{C}$. qPCR primers were designed as explained in Table S1. qPCR was performed using Brightgreen (MasterMix-LR, abm) and $100 \mathrm{ng}$ total cDNA in GAPDH was used as a housekeeping gene in every plate to calculate the $\Delta \mathrm{Ct}$ values.

394 The $\Delta \Delta \mathrm{Ct}$ was calculated with respect to the average $\Delta \mathrm{Ct}$ of colony center (edge vs. center) or $\mathrm{mT}$ (mT vs mTp). 
397 gRNA sequence targeting HPRT1 was obtained from Thermo Fisher (A32060), and then 398 modified following the published protocol (Ran et al., 2013). gRNA was cloned after the U6 promoter sequence into a plasmid containing pCAG-SpCas9-GFP-U6-gRNA

400

401

402

403

404

405

406

407

408

409

410

411

412

413

414

415

416

417

\section{Confocal Imaging}

(Addgene \#79144). $1 \mu \mathrm{g}$ of plasmid was digested using $1 \mu \mathrm{l}$ of Bbs1-HF in 1X cutsmart buffer in a total reaction volume of $50 \mu \mathrm{l}$. This was then run in $1 \%$ agarose gel, and gel extracted following the kit (Zymo D4007).

$10 \mu \mathrm{M}$ of sense and antisense gRNA oligos were added to $1 \mathrm{X}$ T4 DNA ligase reaction buffer with $0.5 \mu \mathrm{l}$ of T4 Polynucleotide Kinase for a final volume of $10 \mu \mathrm{l}$ and annealed in the thermocycler $\left(37^{\circ} \mathrm{C}\right.$ for $30 \mathrm{~min}$, then $95^{\circ} \mathrm{C}$ for $5 \mathrm{~min}$, and ramp down to $25^{\circ} \mathrm{C}$ at $\left.5^{\circ} \mathrm{C} / \mathrm{min}\right)$. The annealed gRNA was ligated into the gel extracted plasmid by adding $50 \mu \mathrm{g}$ of the Bbs1-HF digested plasmid, $1 \mu$ of annealed oligo duplex, and $5 \mu$ of $2 x$ quick ligation buffer for a final volume of $10 \mu \mathrm{l}$. $1 \mu \mathrm{l}$ of quick ligase was then added and the reaction incubated at room temperature for $10 \mathrm{~min} .2 \mu \mathrm{l}$ of this plasmid was added to 50 $\mu$ of Top10 $E$ coli and kept in ice for $5 \mathrm{~min}$. The bacteria were then heat shocked to promote uptake of the plasmid at $42^{\circ} \mathrm{C}$ for 45 seconds before being placed back into ice for 2 min. $250 \mu \mathrm{SOC}$ media was added to the bacteria and incubated in a $37^{\circ} \mathrm{C}$ shaker for $1 \mathrm{~h}$ before being spread onto LB-agar plates with Carbenicillin $(50 \mu \mathrm{g} / \mathrm{ml})$ and incubated overnight at $37^{\circ} \mathrm{C}$. The next day, colonies were picked and grown into LB-broth with Carbenicillin overnight at $37^{\circ} \mathrm{C}$ under shaking. Plasmid was extracted following the kit (Zymo D4210), and concentration was measured using nanodrop. Plasmids were sequenced by Eurofins to check gRNA integration. 
hPSCs were seeded using GD passaging on MG-coated glass bottom dishes (MatTek). added to the culture media for $24 \mathrm{~h}$. Media was aspirated and cells were washed with $1 \mathrm{X}$ PBS, and then fixed with $4 \%$ Paraformaldehyde for 30 min at $37^{\circ} \mathrm{C}$. Cells were washed once and then stored in PBS at $4{ }^{\circ} \mathrm{C}$ until immunostained. Fixed cells were permeabilized with $0.5 \%$ Triton-X100 in PBS for 5 min and then washed in PBST (1X PBS $+0.1 \%$ Tween20) for 3 times for 5 minutes each. Cells were blocked with PBS containing 5\% donkey serum and $0.1 \%$ Tween 20 (blocking buffer). Alexa Fluor 488 conjugated Phalloidin $(4 \mathrm{U} / \mathrm{ml})$ was added to the blocking buffer and incubated with the cells for $1 \mathrm{~h}$ in the dark at room temperature. Dishes were washed with 1X PBST 3 times for 5 minutes each, with $1.43 \mu \mathrm{M}$ DAPI added to the second wash. Cells were stored in 1x PBS while being imaged on Zeiss LSM700 with $63 x / 1.4$ oil objective. Analysis was done using ImageJ with maximum projections of DAPI channel and the middle confocal slice of the Phalloidin labelled F-actin channel of the z-stacks.

\section{CRISPR-Cas9 genome editing of hESCs}

H7-hESC colonies were grown and transfected with the plasmid containing HPRT1-gRNA lipofectamine stem (Invitrogen). Colony dissection was performed 24h after transfection; edge and center samples were collected and plated into mT with $5 \mu \mathrm{M}$ blebbistatin in a changed to $\mathrm{mT}$ without blebbistatin, with culture continuing for another 7-10 days with $\mathrm{mT}$ 
442 cells from the plate and transfer them into PCR tubes. Samples were then vortexed, spun

443 down, and heated at $65^{\circ} \mathrm{C}$ for $10 \mathrm{~min}$ followed by $95^{\circ} \mathrm{C}$ for 5 min to extract DNA. After

444 which the concentration was measured on a nanodrop and 50-200 ng of DNA was used

445 with Phusion-Flash mastermix to PCR amplify the DNA sequence around the HPRT1

446 gRNA target site. The PCR product was run in 1.5\% agarose gel with Ethidium Bromide,

447 and gel extracted following the kit (Zymo D4007). Extracted DNA was then sent for 448 sequencing with Eurofins and analyzed with TIDE analysis software (https://tide.nki.nl/) 449 where CRISPR edge samples were compared to the respective centers.

\section{Lentivirus}

452 H7-hESCs at $~ 80 \%$ confluency were clump passaged using GD and seeded into 96-well

453 MG coated wells. The next day, cell counting was done from a well using accutase 454 mediated single cell dissociation. Lentivirus (LV) (Life Technologies Cat \# A32060) with 455 viral vector containing Pu6-HPRT1(gRNA)-PEFS-GFP was added at multiplicity of infection 456 (MOI) of 10 for each well. LV was added through $\mathrm{mT}$ media containing $8 \mu \mathrm{g} / \mathrm{mL}$ polybrene 457 and the plate was centrifuged at $800 \mathrm{G}$ at room temperature for $1 \mathrm{~h}$ before incubating at $45837^{\circ} \mathrm{C}, 5 \% \mathrm{CO}_{2}$ incubator overnight. The next day, media with lentivirus was removed and 459 replaced with normal $\mathrm{mT}$; $\mathrm{mT}$ was changed every following day and GFP signal was 460 observed over time.

\section{Quantification and Statistical analysis:}

463 All data presented are mean \pm SEM. For statistical analysis between two independent 464 conditions a Student's t-test was performed in Microsoft Excel; for more than two 
465

466

467

468

469

470

471

472

473

474

475

476

477

478

479

480

481

482

483

484

485

487

486 Das, A., Fischer, R.S., Pan, D., and Waterman, C.M. (2016). YAP nuclear localization in

conditions, one-way Anova with Dunnett's multiple comparison post hoc test was performed using GraphPad Prism 9.0 software.

\section{REFERENCES}

Anding, A.L., and Baehrecke, E.H. (2017). Cleaning House: Selective Autophagy of

Organelles. Dev. Cell 41, 10-22.

Bhise, N.S., Wahlin, K.J., Zack, D.J., and Green, J.J. (2013). Evaluating the potential of poly(beta-amino ester) nanoparticles for reprogramming human fibroblasts to become induced pluripotent stem cells. Int. J. Nanomedicine 8, 4641-4658.

Brinkman, E.K., Chen, T., Amendola, M., and Van Steensel, B. (2014). Easy

quantitative assessment of genome editing by sequence trace decomposition. Nucleic Acids Res. 42.

Byrne, S.M., and Church, G.M. (2015). CRISPR-mediated gene targeting of human induced pluripotent stem cells. Curr. Protoc. Stem Cell Biol. 2015, 5A.8.1-5A.8.22.

Chen, K.G., Mallon, B.S., Hamilton, R.S., Kozhich, O.A., Park, K., Hoeppner, D.J., Robey, P.G., and McKay, R.D.G. (2012). Non-colony type monolayer culture of human embryonic stem cells. Stem Cell Res. 9, 237-248.

Chen, K.G., Mallon, B.S., McKay, R.D.G., and Robey, P.G. (2014). Human pluripotent stem cell culture: Considerations for maintenance, expansion, and therapeutics. Cell Stem Cell 14, 13-26. 87 the absence of cell-cell contact is mediated by a filamentous actin-dependent, Myosin 
488 Iland Phospho-YAP-independent pathway during extracellular matrix mechanosensing.

J. Biol. Chem. 291, 6096-6110.

490 Ding, Q., Regan, S.N., Xia, Y., Oostrom, L.A., Cowan, C.A., and Musunuru, K. (2013).

491 Enhanced efficiency of human pluripotent stem cell genome editing through replacing

492 TALENs with CRISPRs. Cell Stem Cell 12, 393-394.

493 Hendel, A., Kildebeck, E.J., Fine, E.J., Clark, J.T., Punjya, N., Sebastiano, V., Bao, G., 494 and Porteus, M.H. (2014). Quantifying genome-editing outcomes at endogenous loci 495 with SMRT sequencing. Cell Rep. 7, 293-305.

496 Hendel, A., Bak, R.O., Clark, J.T., Kennedy, A.B., Ryan, D.E., Roy, S., Steinfeld, I., 497 Lunstad, B.D., Kaiser, R.J., Wilkens, A.B., et al. (2015). Chemically modified guide 498 RNAs enhance CRISPR-Cas genome editing in human primary cells. Nat. Biotechnol. 33, 985-989.

500 Kroemer, G., Mariño, G., and Levine, B. (2010). Autophagy and the Integrated Stress 501 Response. Mol. Cell 40, 280-293.

502 Lombardo, A., Genovese, P., Beausejour, C.M., Colleoni, S., Lee, Y.L., Kim, K.A., 503 Ando, D., Urnov, F.D., Galli, C., Gregory, P.D., et al. (2007). Gene editing in human 504 stem cells using zinc finger nucleases and integrase-defective lentiviral vector delivery. 505 Nat. Biotechnol. 25, 1298-1306.

506 Martin, R.M., Ikeda, K., Cromer, M.K., Uchida, N., Nishimura, T., Romano, R., Tong, 507 A.J., Lemgart, V.T., Camarena, J., Pavel-Dinu, M., et al. (2019). Highly Efficient and 508 Marker-free Genome Editing of Human Pluripotent Stem Cells by CRISPR-Cas9 RNP 509 and AAV6 Donor-Mediated Homologous Recombination. Cell Stem Cell 24, 821-828.e5. 510 Mignon, C., Sodoyer, R., and Werle, B. (2015). Antibiotic-free selection in 
511 biotherapeutics: Now and forever. Pathogens 4, 157-181.

512 Närvä, E., Stubb, A., Guzmán, C., Blomqvist, M., Balboa, D., Lerche, M., Saari, M.,

513 Otonkoski, T., and Ivaska, J. (2017). A Strong Contractile Actin Fence and Large

514 Adhesions Direct Human Pluripotent Colony Morphology and Adhesion. Stem Cell

515 Reports 9, 67-76.

516 Ran, F.A., Hsu, P.D., Wright, J., Agarwala, V., Scott, D.A., and Zhang, F. (2013).

517 Genome engineering using the CRISPR-Cas9 system. Nat. Protoc. 8, 2281-2308.

518 Rosowski, K.A., Mertz, A.F., Norcross, S., Dufresne, E.R., and Horsley, V. (2015).

519 Edges of human embryonic stem cell colonies display distinct mechanical properties

520 and differentiation potential. Sci. Rep. 5.

521 Saha, K., and Jaenisch, R. (2009). Technical Challenges in Using Human Induced

522 Pluripotent Stem Cells to Model Disease. Cell Stem Cell 5, 584-595.

523 Sha, Z., Schnell, H.M., Ruoff, K., and Goldberg, A. (2018). Rapid induction of p62 and

524 GAB ARA PL1 upon proteasome inhibition promotes survival before autophagy

525 activation. J. Cell Biol. 217, 1757-1776.

526 Sharma, P., Yan, F., Doronina, V.A., Escuin-Ordinas, H., Ryan, M.D., and Brown, J.D.

527 (2012). 2A peptides provide distinct solutions to driving stop-carry on translational

528 recoding. Nucleic Acids Res. 40, 3143-3151.

529 Sluch, V.M., Chamling, X., Wenger, C., Duan, Y., Rice, D.S., and Zack, D.J. (2018).

530 Highly efficient scarless knock-in of reporter genes into human and mouse pluripotent

531 stem cells via transient antibiotic selection. PLoS One 13.

532 Steyer, B., Bu, Q., Cory, E., Jiang, K., Duong, S., Sinha, D., Steltzer, S., Gamm, D.,

533 Chang, Q., and Saha, K. (2018). Scarless Genome Editing of Human Pluripotent Stem 
534 Cells via Transient Puromycin Selection. Stem Cell Reports 10, 642-654.

535 Tojkander, S., Gateva, G., and Lappalainen, P. (2012). Actin stress fibers - Assembly,

536 dynamics and biological roles. J. Cell Sci. 125, 1855-1864.

537 Vandermeulen, G., Marie, C., Scherman, D., and Préat, V. (2011). New generation of

538 plasmid backbones devoid of antibiotic resistance marker for gene therapy trials. Mol.

539 Ther. 19, 1942-1949.

540 Yang, L., Guell, M., Byrne, S., Yang, J.L., De Los Angeles, A., Mali, P., Aach, J., Kim-

541 Kiselak, C., Briggs, A.W., Rios, X., et al. (2013). Optimization of scarless human stem

542 cell genome editing. Nucleic Acids Res. 41, 9049-9061.

543 Yen, J., Yin, L., and Cheng, J. (2014). Enhanced non-viral gene delivery to human

544 embryonic stem cells via small molecule-mediated transient alteration of the cell

545 structure. J. Mater. Chem. B 2, 8098-8105. 
Figure 1. hESCs selectively get transfected at the colony edges but not center.

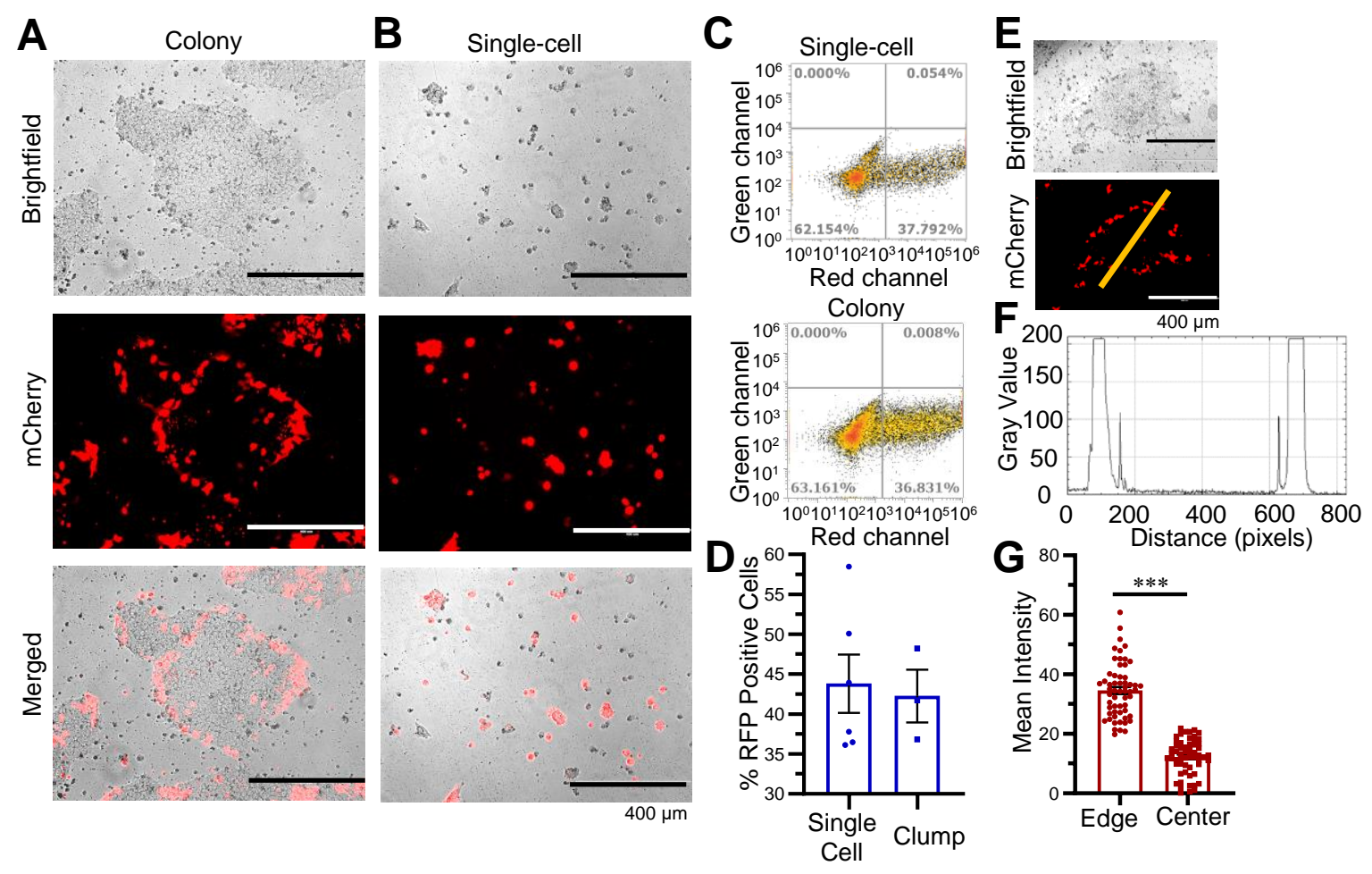


Figure 2. hPSCs at the colony edges are healthier with reduced expression of autophagy genes.

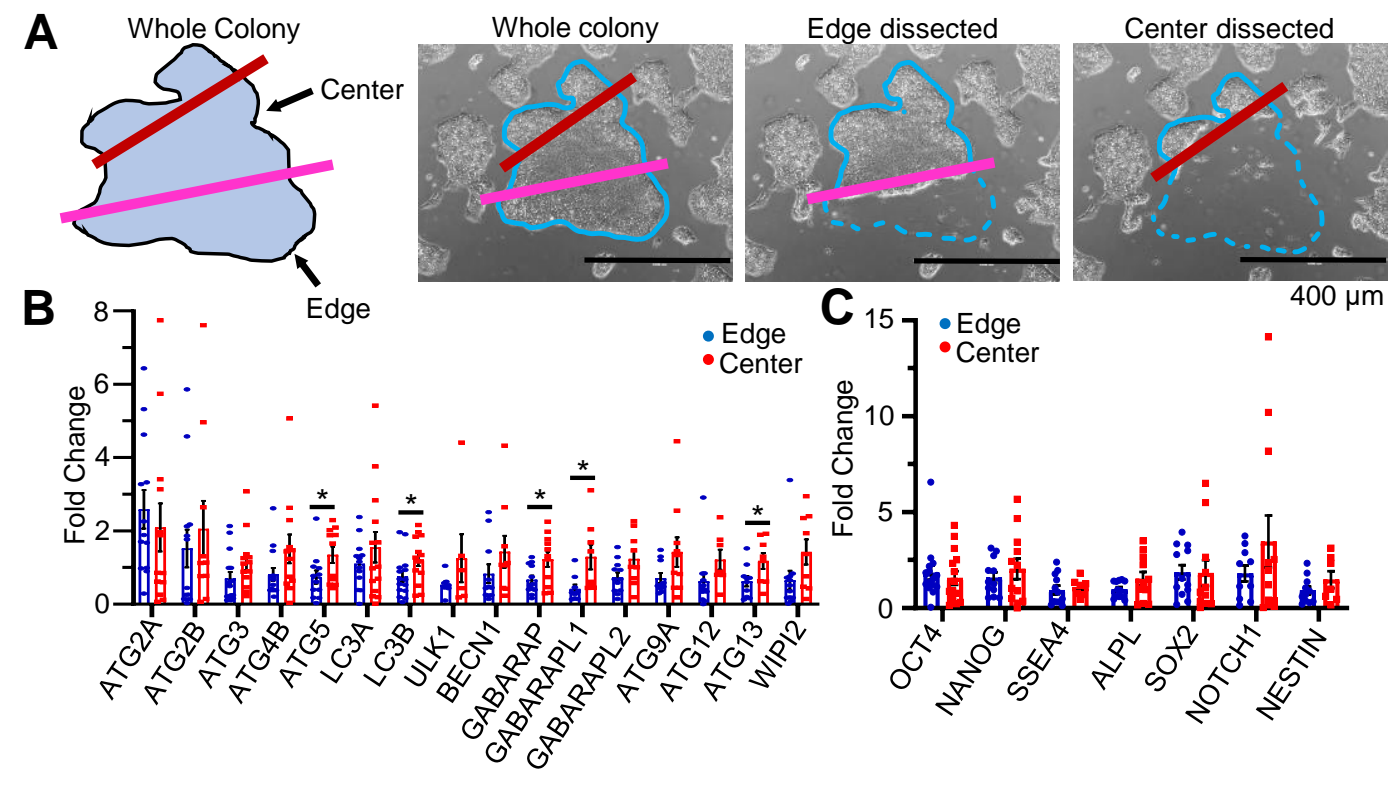


Figure 3. Inhibiting autophagy but not actomyosin contractility decreases transfection efficiency.

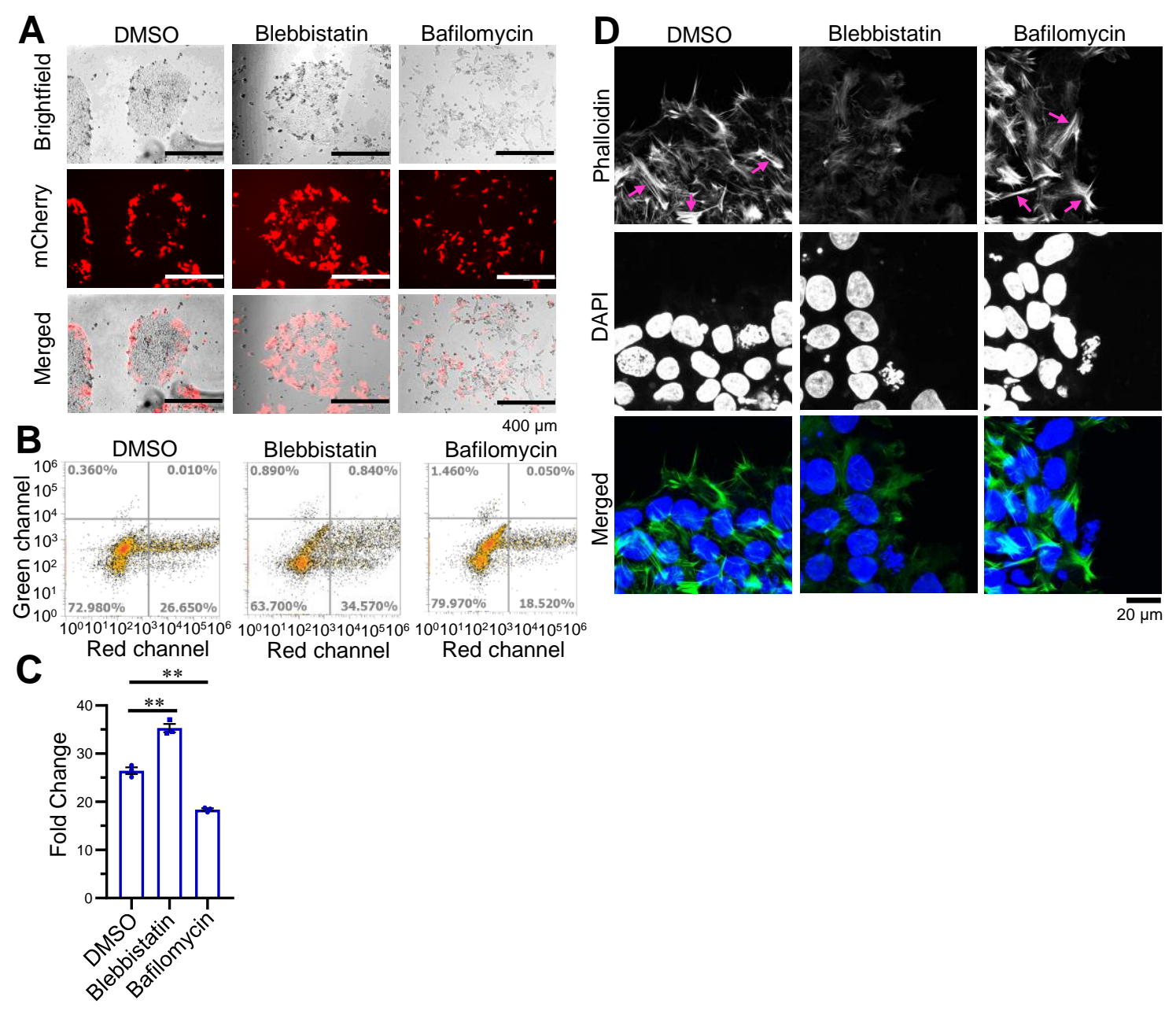


Figure 4. Enhanced CRISPR-Cas9 genome editing at the hPSC colony edges.

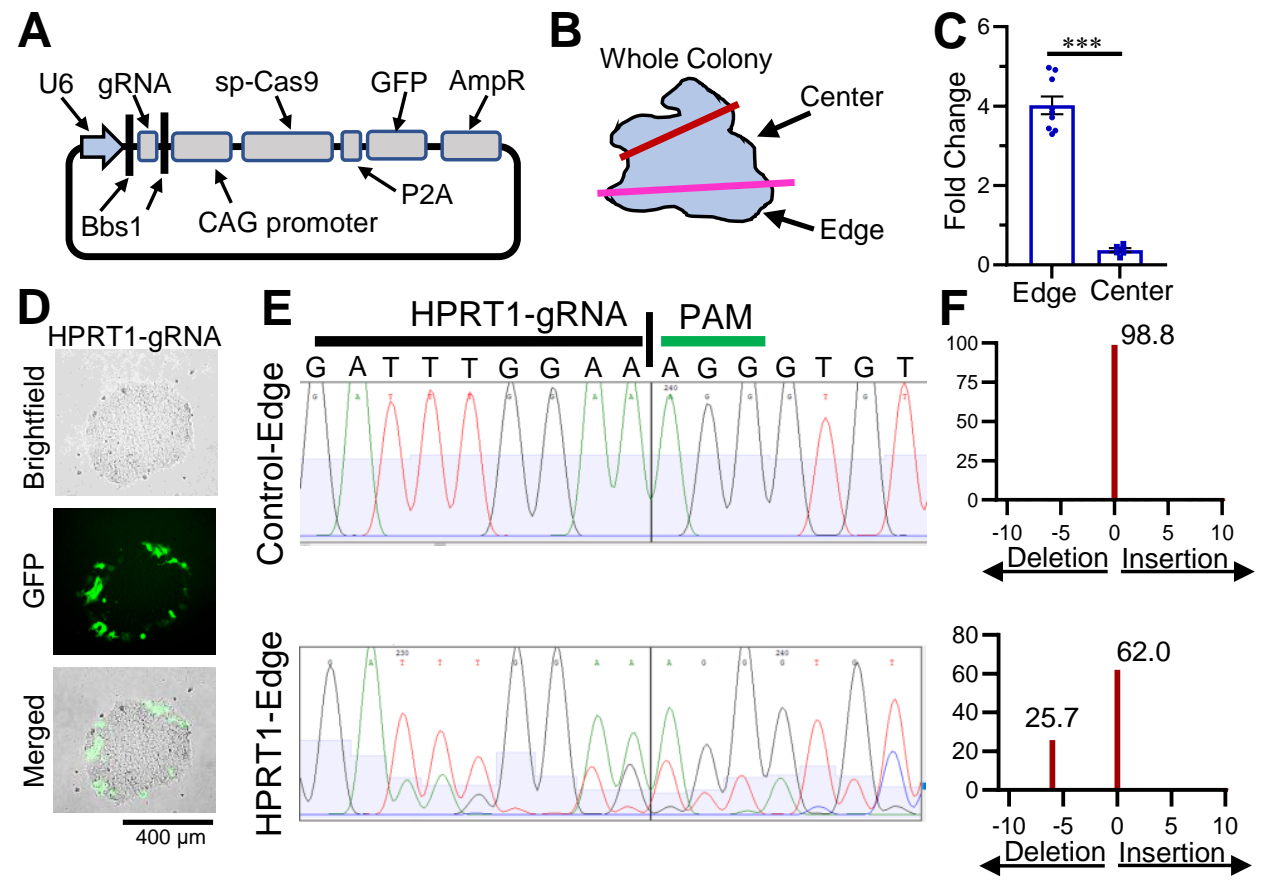




\section{KEY RESOURCES TABLE}

\begin{tabular}{|c|c|c|}
\hline REAGENT or RESOURCE & SOURCE & IDENTIFIER \\
\hline \multicolumn{3}{|l|}{ Antibodies } \\
\hline Alexa Fluor 488 Phalloidin & Invitrogen & Cat\# A12379 \\
\hline \multicolumn{3}{|l|}{ Bacterial and Virus Strains } \\
\hline $\begin{array}{l}\text { Invitrogen }{ }^{\mathrm{TM}} \text { One Shot }{ }^{\mathrm{TM}} \text { TOP10 Chemically Competent } \\
\text { E. coli }\end{array}$ & Fisher Scientific & Cat\# C404003 \\
\hline \multicolumn{3}{|l|}{ Chemicals, Peptides, and Recombinant Proteins } \\
\hline Matrigel & Corning & Cat\# CB40230 \\
\hline Gentle Cell Dissociation Reagent & Stem Cell Technology & Cat\# 7174 \\
\hline mTeSR1 & Stem Cell Technology & Cat\# 85850 \\
\hline mTeSR-Plus & Stem Cell Technololgy & Cat\# 5825 \\
\hline Accutase & Sigma & Cat\# A6964 \\
\hline Blebbistatin & Sigma & Cat\# B0560 \\
\hline Bafilomycin & Sigma & Cat\# B1793 \\
\hline DAPI & Molecular Probes & Cat\# D1206 \\
\hline DMSO & Sigma & Cat\# 276855 \\
\hline Lipofectamine Stem & Invitrogen & Cat\# STEM00003 \\
\hline Optimem & Gibco & Cat\# 31985070 \\
\hline Parafomaldehyde $16 \%$ solution, EM grade & $\begin{array}{l}\text { Electron Microscopy } \\
\text { Sciences }\end{array}$ & Cat\# 15710 \\
\hline Triton-X-100 & Sigma & Cat\# T8787 \\
\hline Donkey Serum & Sigma & Cat\# D9663 \\
\hline Tween-20 & Sigma & Cat\# P9416 \\
\hline Polybrene & Sigma & Cat\# TR-1003-G \\
\hline Bbs1-HF & New England BioLabs & Cat\# R3539S \\
\hline Cutsmart Buffer & New England BioLabs & Cat\# B7204S \\
\hline T4 DNA Ligase Reaction Buffer & New England BioLabs & Cat\# B0202S \\
\hline T4 Polynucleotide Kinase (PNK) & New England BioLabs & Cat\# M0201S \\
\hline Quick Ligation Buffer & New England BioLabs & Cat\# B2200 \\
\hline Quick Ligase & New England BioLabs & Cat\# M2200S \\
\hline SOC media & Fisher Scientific & Cat\# BP974010X5 \\
\hline Carbenicillin & Sigma & Cat\# C1389 \\
\hline LB Broth (Miller) & Sigma & Cat\# L3522 \\
\hline LB broth with agar (Miller) & Sigma & Cat\# L3147 \\
\hline Quick Extraction Buffer & Epicentre & Cat\# QE09050 \\
\hline Phusion Flash Mastermix & Fisher Scientific & Cat\# F548L \\
\hline Agarose & Sigma & Cat\# A9539 \\
\hline Ethidium Bromide & Sigma & Cat\# E1510 \\
\hline \multicolumn{3}{|l|}{ Critical Commercial Assays } \\
\hline RNeasy Mini Kit & Qiagen & Cat\# 74104 \\
\hline $\begin{array}{l}\text { 5x all-in-one RT MasterMix (with AccuRTGenomic DNA } \\
\text { Removal kit) }\end{array}$ & $\begin{array}{l}\text { applied biological } \\
\text { materials }\end{array}$ & Cat\# G492 \\
\hline BrightGreen 2x qPCR MasterMix-Low ROX & $\begin{array}{l}\text { applied biological } \\
\text { materials }\end{array}$ & Cat\# MasterMix-LR \\
\hline ZymoPURE TM Plasmid Miniprep Kit & Zymo & Cat\# D4210 \\
\hline Zymoclean Gel DNA Recovery Kit & Zymo & Cat\# D4007 \\
\hline
\end{tabular}




\begin{tabular}{|c|c|c|}
\hline \multicolumn{3}{|l|}{ Experimental Models: Cell Lines } \\
\hline H7-hESCs, H9-hESCs & $\begin{array}{l}\text { WiCell; Sluch et al., } \\
2018\end{array}$ & \\
\hline EP1-iPSCs & Bhise et al., 2013 & \\
\hline \multicolumn{3}{|l|}{ Oligonucleotides } \\
\hline HPRT1-gRNA-Forward & $\begin{array}{l}\text { ThermoFisher website; } \\
\text { alterations following } \\
\text { Ran et al., } 2013\end{array}$ & $\begin{array}{l}\text { CACCGATTATGCT } \\
\text { GAGGATTTGGAA }\end{array}$ \\
\hline HPRT1-gRNA-Reverse & $\begin{array}{l}\text { ThermoFisher website; } \\
\text { alterations following } \\
\text { Ran et al., } 2013\end{array}$ & $\begin{array}{l}\text { AAACTTCCAAATC } \\
\text { CTCAGCATAATC }\end{array}$ \\
\hline gRNA plasmid sequencing & & $\begin{array}{l}\text { CGCCAGCAACGC } \\
\text { GGCCTTTTTACGG }\end{array}$ \\
\hline HPRT1-PCR-Forward; sequencing & ThermoFisher website & $\begin{array}{l}\text { TACACGTGTGAAC } \\
\text { CAACCCG }\end{array}$ \\
\hline HPRT1-PCR-Reverse & ThermoFisher website & $\begin{array}{l}\text { GTAAGGCCCTCCT } \\
\text { CTTTTATTT }\end{array}$ \\
\hline Primers for qPCR & $\begin{array}{l}\text { Supplemental Table } \\
\text { S1 }\end{array}$ & \\
\hline \multicolumn{3}{|l|}{ Recombinant DNA } \\
\hline pCAG-mCherry plasmid & Addgene & Cat\# 108685 \\
\hline pCAG-SpCas9-P2A-GFP-U6-gRNA & Addgene & Cat\# 79144 \\
\hline pCAG-SpCas9-P2A-GFP-U6-HPRT1 & This paper & N/A \\
\hline $\begin{array}{l}\text { LentiArray }^{\mathrm{TM}} \text { CRISPR Positive Control Lentivirus, human } \\
\text { HPRT, with GFP (Pu6-HPRT1-PEFs-GFP) }\end{array}$ & $\begin{array}{l}\text { ThermoFisher (Life } \\
\text { Technologies) }\end{array}$ & Cat\# A32060 \\
\hline \multicolumn{3}{|l|}{ Software and Algorithms } \\
\hline ImageJ & $\mathrm{NIH}$ & \\
\hline Attune NxT Software & ThermoFisher & \\
\hline Prism version 9 & GraphPad & \\
\hline Zen Microscope Software & Zeiss & \\
\hline Genescript & $\begin{array}{l}\text { https://www.genscript. } \\
\text { com/ }\end{array}$ & \\
\hline Primer3 & https://primer3.ut.ee/ & \\
\hline PrimerBank & $\begin{array}{l}\text { https://pga.mgh.harvar } \\
\text { d.edu/primerbank/ }\end{array}$ & \\
\hline TIDE analysis software & https://tide.nki.nl/ & \\
\hline
\end{tabular}

